\title{
The Photo-Injector Option for CLIC: Past Experiments and Future Developments
}

\author{
H.H. Braun, E. Chevallay, S. Hutchins, P. Legros, G. Suberlucq, \\ H.Trautner, CERN, Geneva, Switzerland, \\ I.N. Ross, Central Laser Facility, RAL, Didcot, U.K., \\ E. Bente, University of Strathclyde, Institute of Photonics, Glasgow, U.K.
}

\begin{abstract}
The Compact Linear Collider (CLIC) drive beam requires a long bunch train $(92 \mu \mathrm{s})$ consisting of 42880 bunches with a bunch charge of $17.5 \mathrm{nC}$ in a bunch length of less than 20 ps. This train might be produced by an RFphoto-injector equipped with a Cs-Te cathode. After a short review of experience with such cathodes in the present CLIC Test Facility (CTF2), mainly focused on the production of short trains of high-charge bunches, we will present the scheme foreseen for CLIC and CTF3. The laser will be a diode-pumped MOPA (Master Oscillator Power Amplifier), operating at a repetition rate of $469 \mathrm{MHz}$ for CLIC and $1.5 \mathrm{GHz}$ for CTF3. The specific requirements of an RF-gun for this high-current operation are discussed. New experimental results on the photocathode lifetime at high average current are summarized.
\end{abstract}

\section{PAST EXPERIENCES IN CTF2}

Laser driven photo-injectors have been used in the CTF2 since its inception. The CTF2 laser system is a Chirped Pulse Amplification system using a $250 \mathrm{MHz}$ modelocked oscillator, one regenerative and two single pass amplifiers. The near-infrared light is frequency quadrupled to UV $(\lambda=262 \mathrm{~nm})$ which is then split into 48 pulses, each with a duration of $10 \mathrm{ps}(\mathrm{FWHH})$. These are sent to the cathode of the drive beam RF gun. The residual green light is converted to produce a single UV pulse which illuminates the cathode of the probe beam RF gun [1].

For the CTF2 drive beam, an RF gun with a CsTe cathode is routinely used to produce a bunch train with bunchcharges of up to $15 \mathrm{nC}$. The field gradient on the cathode surface is $100 \mathrm{MV} / \mathrm{m}$. CsTe cathodes offer quantum efficiencies $(\mathrm{QE})$ of over $1.5 \%$ with lifetimes varying from at least some weeks to typically months. Since the production of UV light poses no major problems, this kind of cathode becomes more and more attractive.

The cathodes for CTF2 are produced in a dedicated laboratory, where their QE is measured in a DC gun. They are then brought under ultra-high-vacuum to the accelerator. The cathode for the probe beam is $\mathrm{CsI}+\mathrm{Ge}$, which can be transferred in air [2].

\section{CLIC DRIVE BEAM REQUIREMENTS}

For efficient RF production, CLIC requires a long train of electron bunches with high charge. Table 1 shows some
Table 1: Drive Beam Parameters

\begin{tabular}{|c|c|c|c|}
\hline Pulse charge & $\begin{array}{l}\text { Unit } \\
\mathrm{nC}\end{array}$ & $\begin{array}{c}\text { CTF3 } \\
2.4\end{array}$ & $\begin{array}{c}\text { CLIC } 3 \mathrm{TeV} \\
17.5\end{array}$ \\
\hline Pulse width (FWHH) & ps & 10 & 12 \\
\hline Peak current & A & 240 & 1450 \\
\hline Number of pulses & - & 2145 & 42880 \\
\hline$t$ between pulses & ns & 0.666 & 2.13 \\
\hline Charge stability & $\% \mathrm{rms}$ & $<0.5$ & 0.1 \\
\hline Train duration & $\mu \mathrm{s}$ & 1.4 & 92 \\
\hline Train charge & $\mu \mathrm{C}$ & 5.2 & 750 \\
\hline Repetition rate & $\mathrm{Hz}$ & 5 & 100 \\
\hline Mean current & $\mathrm{mA}$ & 0.026 & 75 \\
\hline $\mathrm{QE}_{\min }$ at $\lambda_{\text {laser }}$ & $\%$ & 1.5 & 1.5 \\
\hline$\tau_{\min }$ at $\mathrm{QE}_{\min }$ & $\mathrm{h}$ & 100 & 24 \\
\hline Shots during $\tau_{\min }$ & $\mathrm{x} 10^{9}$ & 3.9 & 371 \\
\hline Produced charge & $\mathrm{C}$ & 10 & $6.5 \mathrm{k}$ \\
\hline $\bar{P}_{\text {laser,cath }}$ & $\mathrm{W}$ & 0.008 & 23.3 \\
\hline
\end{tabular}

of the CLIC and CTF3 drive beam injector parameters. The challenges are to build a laser capable of delivering adequate light to the RF gun, and to make photocathodes that are able to produce the required electron beams.

\section{MOTIVATION FOR A DRIVE BEAM PHOTO-INJECTOR}

Although the reference design of CTF3 and CLIC assumes a thermionic drive-beam injector [3], an option for a photo-injector is kept open because of its advantages:

- flexibility in manipulating the time structure of the beam because laser beams can be switched faster than electron beams in a thermionic high-voltage gun. This is important for minimizing transient beam-loading in the CLIC main beam [4].

- no problems with parasite charges in the supposedly empty RF buckets (in the drive-beam linac only every second bucket is populated, however it is difficult to get zero charge in the "empty" buckets with a conventional bunching system). This unwanted charge can cause considerable radiation problems and efficiency loss. - smaller transverse and longitudinal emittance and thus easier beam transport and bunch length manipulation. - Smaller longitudinal emittance and therefore easier bunch length manipulation in the downstream systems. - no low-energy tails at the end of the injector. 


\section{CTF3 RF GUN DESIGN}

The design of the $3 \mathrm{GHz} \mathrm{RF}$ gun for the drive beam is based on the existing CTF2 drive-beam gun [5]. However, to cope with the heavy beam loading of CTF3, the $\mathrm{RF}$ coupling factor $\beta$ has to be increased to $\beta=2.9$. This will allow an equilibrium between the power taken by the beam $\left(\mathrm{E}_{\text {beam }}=5.6 \mathrm{MeV}, \mathrm{I}_{\text {beam }}=3.5 \mathrm{~A}\right)$, the ohmic losses in the cavity $(\mathrm{Q}=13000)$ and the input power provided by the klystron (30 MW).

\section{THE LASER}

The laser system for the photo-injector has a number of challenging operating parameters, namely the necessity to produce a long $(92 \mu \mathrm{s})$ pulse train with high average power with low intensity jitter, the synchronization to the

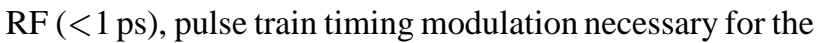
RF manipulation of the electron beam in the CLIC scheme [3] and high operational reliability. Other parameters are mentioned in Table 1.

A feasibility study [6] concluded that the operating parameters could best be achieved with an all solid-state system consisting of a diode-pumped modelocked oscillator and power amplifier system that operates around $1 \mu \mathrm{m}$, which is frequency quadrupled. Nd:YLF operating at $1047 \mathrm{~nm}$ was selected for the laser material as it offers a combination of several important properties: These were the high extraction efficiency, good optical quality in large pieces and low thermal distortion. Tests are underway to improve the conversion efficiency of $10 \%$ from IR to UV, since this directly affects the diode power needed.

\subsection{The Oscillator}

The laser oscillator will be passively modelocked using a saturable Bragg reflector (SBR) providing a modelocked ps pulse train with the same reliability as a cw laser. The greater the power available from the oscillator system, the less complex the amplifier system can be, therefore work has started on a high-power modelocked oscillator. For the laser oscillator, the following set-up is being prepared for tests (see Fig. 1). The laser crystal is a $12 \mathrm{~mm}$ long brick

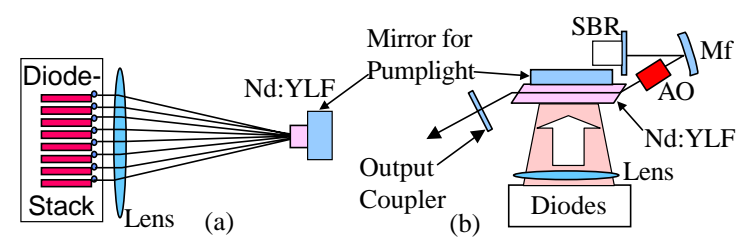

Figure 1: Schematic overview of the laser oscillator setup. (a) Side view of the pump geometry. (b) Top view of the oscillator (Mf - curved folding mirror, AO - acousto-optic modulator).

with Brewster faces. The crystal is side-pumped by a stack of 8 fast-axis collimated diode arrays that can produce a total of $300 \mathrm{~W}$. The diode arrays will be operated in a quasi- cw mode for approximately $200 \mu$ s. The resonator will be a three-mirror folded cavity with a curved fold mirror to reduce the modesize on the SBR. Since the repetition rate of the modelocked train is determined by the oscillator cavity length, the laser pulses can be synchronized to an external clock by active control of the cavity length using a piezomounted mirror. A faster way of length modulation of the cavity might be required in order to be able to fully operate the modelocked laser in quasi-cw mode. This will be part of the investigation. In this way it is hoped that over $50 \mathrm{~W}$ of average output power over the macro-burst can be produced.

\subsection{The Amplifier Chain}

It is planned to install three amplifiers, as shown in Fig. 2. The first two operate in double pass, the third in

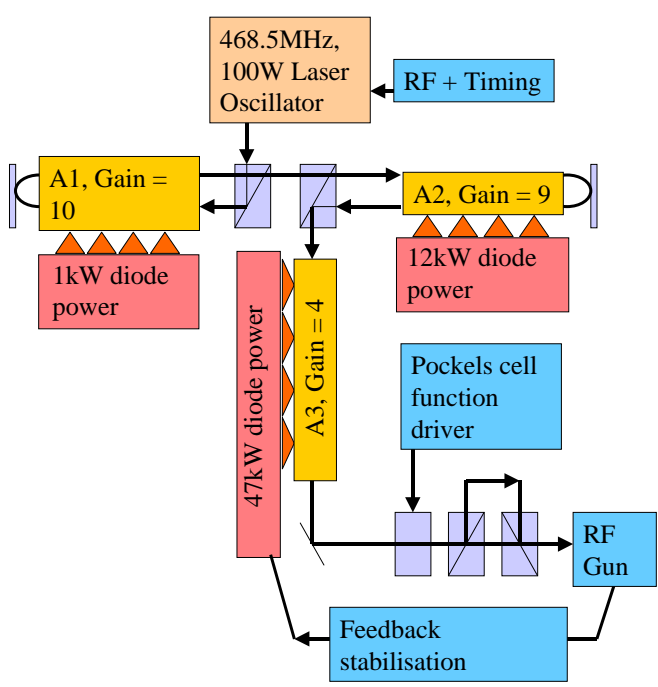

Figure 2: Amplifier chain for CLIC

a single pass and saturated mode, which efficiently extracts the absorbed pump power. In total $59 \mathrm{~kW}$ of diode pump power will be installed [6]. The basic design of the single amplifier heads is a 5-fold symmetrically side pumped rod configuration, with a Nd:YLF rod of $1 \mathrm{~cm}$ diameter, surrounded by water and a silica tube, which reflects pump light not absorbed back into the rod. This should give an absorption efficiency of $85 \%$. A test amplifier is currently assembled at RAL. With $4.5 \mathrm{~kW}$ pump power operated in quasi-cw mode, this amplifier head will be used to verify several important aspects of the design [6].

\section{CATHODES}

\subsection{High average current test}

Since the laser for the CLIC drive beam seems feasible, the question remained if the CsTe cathodes would sustain the production of a high-average-current electron beam, 
under illumination with a high-power-laser beam. Photocathode tests in an RF gun would require the laser system that is currently under development [6], so the test was conducted in a DC gun [7]. The laser was an intracavity frequency quadrupled Nd:YLF. Table 2 summarizes its important parameters and the results of the test. The current delivered by the power supply, the laser power and the vacuum level were measured in real time, the laser spot size on the cathode was controlled in regular intervals. Fig. 3 shows the behaviour of one of the best cathodes tested. The

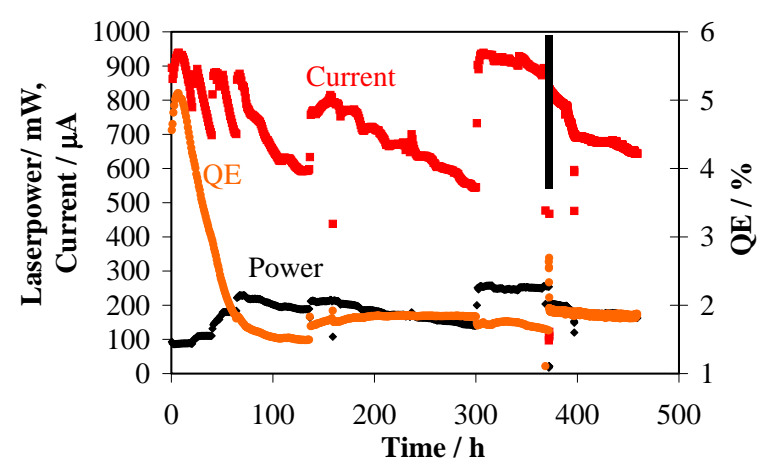

Figure 3: Behaviour of a CsTe cathode under high average current; total produced charge $1.22 \mathrm{kC}$.

laser spot size on the cathode was $37 \mathrm{~mm}^{2}$ during this test. The slight increase in QE in the first hours is probably due to the slow formation of the correct stoichiometric ratio. With an increase of laser power, the QE increased again slightly. This is a hint that the laser light cleans the cathode from contamination by the rest gas. We produced a total charge of $1 \mathrm{kC}$ in this configuration at an average current of $751 \mu \mathrm{A}(750 \mathrm{nC}$ at $1 \mathrm{kHz})$. We stopped this test after $370 \mathrm{~h}$.

Table 2: Results of the high average current test

\begin{tabular}{|c|c|c|}
\hline & Unit & High Q Test \\
\hline Pulse charge & $\mathrm{nC}$ & 950 \\
\hline Pulse width (FWHH) & $\mathrm{ns}$ & 100 \\
\hline Peak current & $\mathrm{A}$ & 7.5 \\
\hline Repetition rate & $\mathrm{Hz}$ & 1000 \\
\hline Mean current & $\mathrm{mA}$ & 0.95 \\
\hline$\tau_{\min }$ at $\mathrm{QE}_{\min }$ & $\mathrm{h}$ & 460 \\
\hline Shots during $\tau_{\min }$ & X $10^{9}$ & 4 \\
\hline Total produced charge & $\mathrm{C}$ & 1220 \\
\hline $\bar{P}_{\text {laser,cath }}$ & $\mathrm{W}$ & 0.3 \\
\hline
\end{tabular}

\subsection{High current density test}

The same cathode was then used to conduct a high current density test, during which the laser was focused to a spot size of $3.34 \mathrm{~mm}^{2}$. The cathode produced $220 \mathrm{C}$ with an average current of $701 \mu \mathrm{A}$, and thus delivered an average current density of $21 \frac{\mathrm{mA}}{\mathrm{cm}^{2}}$. The behaviour under this high flux is shown in Fig. 3 after the line at 370 h. Assuming that the current density obtained is the maximum for this cathode material, a cathode for CLIC would require a diameter of $2.1 \mathrm{~cm}$, which is feasible for a $469 \mathrm{MHz} \mathrm{RF}$ gun.

\section{FUTURE DEVELOPMENTS}

The production of cathodes for relatively relaxed applications like the CTF 3 probe beam, may not need an installation with online QE measurement, heating and cleaning. It was already shown in the past that an evaporation of $10 \mathrm{~nm}$ of Te and $12 \mathrm{~nm}$ of Cs produced cathodes with a QE between $0.25 \%$ and $1 \%$. Therefore, a preparation chamber attached to the CTF2 probe beam gun is under test. Lately, a cathode based on a $2 \mathrm{~nm}$ thin Te layer and $6.5 \mathrm{~nm}$ of Cs was produced, exhibiting a QE of $12 \%$. This gives hints about the thickness of CsTe from which electrons can escape into the vacuum.

Control systems are under development which will modulate the diode current to achieve the $0.3 \%$ stability of the electron bunch train, and high bandwidth detectors capable of monitoring the microbunch intensity are being investigated. The pulse train timing modulation system is also being prepared at CERN.

\section{CONCLUSIONS}

The feasibility of a photo-injector for CLIC has been demonstrated. The proposed laser chain consists of proven technology, with some technical challenges. Although the CLIC value for beam current has not yet been demonstrated, the cathodes have shown that they are capable of delivering a high current density, which is crucial for a CLIC drive-beam photo-injector.

\section{REFERENCES}

[1] S.C.Hutchins, "CTF2 Laser and Optical System”, CERN/PS CTF Tech Note 2000-09

[2] E. Chevallay, S. Hutchins, P. Legros, G. Suberlucq, H. Trautner, "Studies and Production of Photocathodes for High Intensity Electron Beams", CERN CLIC Note 449

[3] The CLIC Study Team (ed. R. Corsini), "The CLIC RF power source : a novel scheme of two-beam acceleration for e-/e+ linear colliders", CERN yellow report, 99-06

[4] D. Schulte, I. Syratchev, "Beam Loading Compensation in the main Linac of CLIC”, Proc. of XX Int. Linac Conf., Monterey, 2000

[5] R. Bossart, H.H. Braun, M. Dehler, J.C. Godot, "A $3 \mathrm{GHz}$ Photoelectron Gun for High Beam Intensity", Proc. FEL Conf., New York 1995

[6] I.N.Ross, "Feasibility Study for the CERN CLIC PhotoInjector Laser System", Central Laser Facility, RAL, CLIC note 462

[7] E. Chevallay, G. Suberlucq, H. Trautner, "Production of a high average current electron beam with CsTe photocathodes", CTF3 Note 020 